\title{
MedienPädagogik
}

Zeitschrift für Theorie und Praxis der Medienbildung

Jahrbuch Medienpädagogik 2.

Zweitveröffentlichung aus: Jahrbuch Medienpädagogik 2. (2001) Opladen: Leske + Budrich. Herausgegeben von Ben Bachmair, Dieter Spanhel und Claudia de Witt.

\section{Medienpädagogik des Pragmatismus}

\author{
Karl-Hermann Schäfer
}

\section{Einleitung}

Grundprobleme der Medienpädagogik sind Grundfragen, die beantwortet müssen, damit eine Gesamtkonzeption der Medienpädagogik entwickelt werden kann. Gegenstandsfeld der Medienpädagogik ist der Zusammenhang von Heranwachsenden und Medien, der durch Lehrer, Erzieher, Fort- und Weiterbildner vermittelt wird. Dieser Zusammenhang oder dieses Gefüge von Lernenden und Medien ist in umgreifende gesellschaftliche Situationen und Institutionen eingebettet und wird sowohl von der Allgemeinen Medienpädagogik als auch von den spezifischen Medienpädagogiken ausgelegt.

Bereits in diesen einleitenden Passagen soll sichtbar werden, daß Medienrezeption und Medienproduktion als die beiden einander ergänzenden Seiten eines einheitlichen Zusammenhangs betrachtet werden müssen, der in der Allgemeinen Medienpädagogik und in den spezifischen Medienpädagogiken des Pragmatismus seinen angemessenen Ausdruck findet.

\subsection{Medienpädagogik als Medienmethodik}

Im Kontext des Pragmatismus ist es nicht sinnvoll, inhaltliche und methodische Probleme scharf voneinander zu „trennen“, um ihre Lösung sodann unterschiedlichen wissenschaftlichen Disziplinen zuzuweisen. Es liegt in der Logik einer solchen Trennung, die inhaltlichen Probleme der jeweiligen Wissenschaft im engeren Sinne und die methodischen Probleme einer sogenannten Methodik zuzuweisen. Im Gegensatz zu einem solchen Verfahren werden die inhaltlichen und die methodischen Probleme vom Pragmatismus als zu „unterscheidende“, aber nicht als zu „trennende“ Aspekte eines einheitlichen, praktischen Zusammenhangs betrachtet.

Im übrigen ist davon auszugehen, daß Probleme nur gelöst werden können, wenn die Problemlösung als ein aktiver „Prozeß“ begriffen wird, der immer schon eine methodische Strukturierung aufweist, bei der entschieden werden muß, welcher Gesichtspunkt „zuerst“ und welcher andere Aspekt „danach“ behandelt wird. Der „Prozeß“ der Problemlösung bezeichnet infol- 
gedessen einen „Weg“ von griechisch mét-hodos, der zur Erreichung eines Ziels zu verfolgende „Weg“ (hodos), der gegangen wird und der aber immer schon Inhalte und damit Ziel vorstellungen enthält.

Was nun das Problemfeld „Medienpädagogik als Medienmethodik“ betrifft, so schlage ich vor, von John Deweys Methodik-Konzeption auszugehen und Methodik als einen aktiven „problemlösenden Prozeß“ zu betrachten, den Dewey „inquiry process“ nennt und der überaus differenziert und problembewußt in John Deweys „Logik“ des Pragmatismus dargestellt wird (vgl. Dewey 1986; vgl. de Witt 2000). Dieser „aktive“ problemlösende Prozeß bezeichnet das aktive Moment der Medienrezeption und der Medienproduktion. Diese methodische und methodologische Grundorientierung, welche die inhaltlichen und zielorientierten Vorstellungen enthält, ist implizit und explizit in allen 37 Bänden von John Deweys Gesamtwerk präsent. Darüber hinaus ist es sinnvoll, John Deweys Methodik-Konzeption gemäß den Prinzipien von John Deweys Lehrer Charles Sanders Peirce und nach den Grundsätzen seines Schülers Charles William Morris weiter aus zu differenzieren. In diesem Prozeß der Ausdifferenzierung muß den Kategorien von Charles William Morris „Syntaktik“, „Semantik“ und „Pragmatik“ eine herausragende Bedeutung beigemessen werden, weil sie methodische Teildisziplinen darstellen, die sich in den Kultur-, Sozial- und Naturwissenschaften der englisch-sprachigen Welt und zum Teil auch in Europa bereits bewährt haben (vgl. Nöth 2000).

\subsection{Erlebnisorientierung als eine mögliche Voraussetzung oder als eine Vorstufe der aktiven Medienrezeption}

Damit besser verständlich wird, was ein Medienerlebnis ist, schlüpfen wir in die Rolle der Schüler und schauen uns einen Spielfilm an. Relevant ist in diesem Zusammenhang, daß wir das Medienerlebnis mit den Schülern teilen, ihnen gleichsam über die Schulter schauen, den Film aus „ihrer Perspektive“ betrachten und die Position eines „teilnehmenden Beobachters" einnehmen. Es wird dunkel, der Film beginnt, und wir finden uns wieder in einer anderen Wirklichkeit. Wir vergessen die Leinwand, und daß wir sich bewegende Bilder sehen. Wir hören Geräusche und Dialoge, die von Musik begleitet werden. Wir steigen ein in ein Geschehen, das sich unseren Augen und Ohren darbietet. Am Ende unseres Blickes sind wir bei Helden und Opfern, die kämpfen, leiden, siegen oder untergehen. Wir sind unmittelbar bei den Menschen, deren Geschichten wir unmittelbar visuell und auditiv miterleben und deren Wirklichkeit während des Filmgenusses unsere Wirklichkeit ist. Auf magische Weise werden wir in eine andere Wirklichkeit hineingezogen, so daß wir bei unseren Helden sind.

Dieser Zusammenhang, den wir erleben, ist ein unmittelbarer Zusammenhang, weil uns während dieses Medienerlebnisses all die vermittelten Komponenten dieses Medienerlebnisses weitgehend unbewußt bleiben. Uns ist nicht bewußt, daß vermittelnde Komponenten dieses Medienerlebnisses 
wie erlebnishaftes Sehen, Hören, ein intuitives Verständnis von erlebten Handlungen und ein spezifisches Medium nötig sind, um dieses Einheitserlebnis möglich zu machen.

Auf reflexive Denkprozesse, die den Schüler-Film-Zusammenhang objektivieren können, verzichten wir. Die einsetzende Reflexion würde den unmittelbaren Erlebniszusammenhang zerstören. Die Reflexion würde den Erlebniszusammenhang auseinander brechen lassen.

Solange der Film läuft, sind wir in das Filmgeschehen eingebunden. Wir verhalten uns unmittelbar proflexiv. In unserer Vorstellung „wenden wir uns“ den Akteuren der Filmhandlung „zu“. „Proflexion“ nennt der Philosoph und Pädagoge Franz Fischer die Bewegung, in der und durch die wir uns Personen und Sachverhalten „zuwenden“. In dieser vorstellungsmäßigen „Vorwärtsbewegung der Zuwendung“, in dieser „Pro-Flexion“, ist unser erlebendes Bewußtsein bei den Filmakteuren dort draußen. In diesem proflexiven Erlebnis bleibt unser sinnliches Bewußtsein zunächst einmal gefesselt an das Ende unseres Blickes, das unsere Aufmerksamkeit vollständig erfüllt.

Schaubild 1: Erlebnisorientierung als Vorstufe der aktiven Medienrezeption: Das proflexive Medienerlebnis als unmittelbarer Zusammenhang von dargestellter Wirklichkeit, Medium und Medienrezipienten

\section{Das Medienerlebnis als Vorstufe der Medienpädagogik}

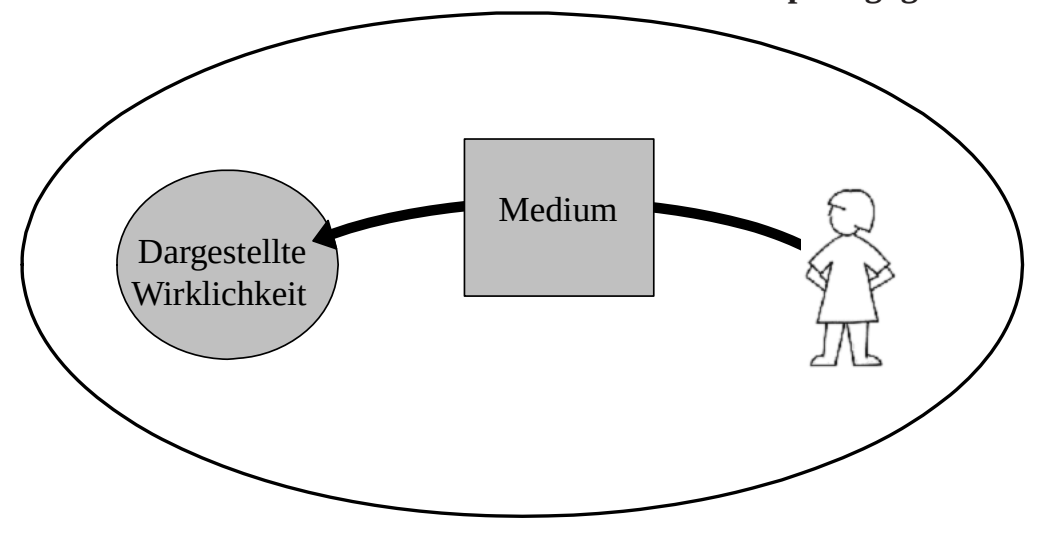

Was für den Film gilt, hat die gleiche oder eine vergleichbare Bedeutung für andere Medien. Wenn wir ein Buch lesen, das uns fesselt, tauchen wir ein in die Welt, die ein Buch sichtbar werden läßt. Auch Bücher sind Medien. Es ist eine Tatsache, daß Bücher zum Erlebnis werden können. Nicht alle Bücher haben diese Wirkung auf uns, aber - und das ist entscheidend - es ist möglich! Dies gilt nicht nur für Romane, es gilt auch für wissenschaftliche Bücher, die für uns relevant sind. Diesem Zusammenhang zwischen den lesenden Schülern und den Büchern geht die Buchpädagogik nach, die in den Umkreis der spezifischen Medienpädagogiken gehört. Natürlich hat die Buch- 
Pädagogik nicht nur die erlebnishaften Zusammenhänge zu erforschen. Hinzu kommen die syntaktischen, die semantischen und die pragmatischen Dimensionen, die später erläutert werden.

Die Hörfunkpädagogik erforscht anders strukturierte Zusammenhänge, die zwischen den Edukanden und Rundfunksendungen ablaufen. Wenn wir in die Welt der Musik oder in die fremde Wirklichkeit eines Hörspiels „eintauchen“, entstehen in uns erfüllte Klangräume und dargestellte Wirklichkeiten unseres intentionalen Hörens, die ebenso Erlebniszusammenhänge darstellen. Ähnliches gilt für die Fernsehpädagogik, die unterschiedliche Sparten des Fernsehens erforscht, analysiert und interpretiert. Fernsehspiele, Sportveranstaltungen, Musiksendungen und andere Sparten können Begeisterungsstürme auslösen und Erlebniszusammenhänge stiften.

Von den spezifischen Medienpädagogiken spielt die Computerpädagogik eine herausragende Rolle. Der Computer ist ein universales Medium, das andere Medien in sich aufnimmt und sie integriert. Der PC als eine universale und elektronische Maschine der Datenverarbeitung fungiert als ein Medium, das Seh- und Hörerlebnisse mannigfaltiger Art möglich macht. Der Computer kann Geschichten erzählen, die wir selbst erfunden haben, kann Musik-CDs zum Klingen bringen, Filme aller Art als DVDs zeigen, kann Abenteuerspiele ermöglichen und kann uns über die Brücke der CDs in Literatur in philosophische und pädagogische Systeme „eintauchen“ lassen. Der Computer kann uns im Internet neue vernetzte Kommunikationsräume erschließen und sie uns erleben lassen.

Alle diese emotionalen Ereignisse weisen auf einen Sachverhalt hin. Medien wollen - wo immer dies möglich und sinnvoll ist - zunächst einmal „erlebt“ werden. Die Erlebnisdimension ist daher eine relevante Voraussetzung oder eine Vorstufe einer aktiven Medienrezeption, der eine bildende Bedeutung zugemessen werden soll.

\section{Drei Stufen der aktiven Medienrezeption}

\subsection{Die Syntaktik als die erste Stufe der aktiven Medienrezeption}

Medienerlebnisse sind zunächst immer je spezifische Erlebnisse von Individuen, die in unserem Zusammenhang Schüler und Lehrer, Studierende und Weiterbildner sein können. Zum anderen sind Erlebnisse auch immer an spezifische Medien gebunden. Insofern ist es sinnvoll bei der Interpretation von Erlebniszusammenhängen an die spezifischen Medienpädagogiken zu denken. Verallgemeinern wir diesen Zusammenhang, dann kommen wir zu dem Ergebnis, daß die Vorstufe der Allgemeinen Medienpädagogik ein sinnlichpraktischer Erlebniszusammenhang ist, der die Edukanden mit den Medien unmittelbar verbindet, wobei dem Lehrer, dem Erzieher oder dem Weiterbildner die Aufgabe zugewiesen wird, diesen pädagogisch relevanten Erlebniszusammenhang zu ermöglichen. 
Wir schreiten nun weiter voran, um von der Vorstufe des sinnlichpraktischen Erlebniszusammenhangs zur ersten Stufe des reflexiven Theorie-Zusammenhangs zu gelangen. Damit dies gelingt, ist ein Standortwechsel oder ein Perspektivenwechsel erforderlich. Es ist ein besonderes Kennzeichen von Erlebnissen, daß sie intentional ausgerichtet sind. Intentionalität meint die Gerichtetheit, die Zielgerichtetheit und damit den Tatbestand, daß Erlebnisse auf eine Erlebniswirklichkeit hin ausgerichtet sind, die von Medien dargestellt werden. Nun sind zwei Arten von Intentionalität, die „intentio directa“ und die „intentio reflexiva“ zu unterscheiden. Mit Fischer grenzen wir im folgenden die intentio directa als vorwärts ausgerichtete Proflexion von der intentio reflexiva als der rückwärts ausgerichteten Reflexion ab. Die Proflexion als intentio directa steht also der Reflexion als der intentio reflexiva gegenüber.

Die unmittelbare Erlebniseinheit von Edukandus und Medienwirklichkeit zerbricht, wenn die Reflexion einsetzt und die Proflexion damit zunächst einmal verdrängt. Dewey spricht in diesem Zusammenhang davon, daß die unmittelbar erlebte Einheit der Erfahrung, die den Edukandus mit den Medien verbindet, „auseinander bricht“. Die einheitliche Ganzheit des erlebten Erfahrungszusammenhangs „,is broken up“ und nun werden die einzelnen Komponenten der vormaligen Erlebniseinheit sichtbar (Dewey 1983, S. 349-396). Dort, wo dieser kontinuierliche Zusammenhang „zerbricht“, werden die einzelnen Bestandteile dieses Zusammenhangs sichtbar und der Bereich der wissenschaftlichen Theorie entsteht.

Schaubild 2: Die Reflexion läßt die einzelnen Komponenten der Medienpraxis auseinander treten und die Medienpraxis als Erlebniszusammenhang bricht auseinander

Die „Wirkung“ der Reflexion

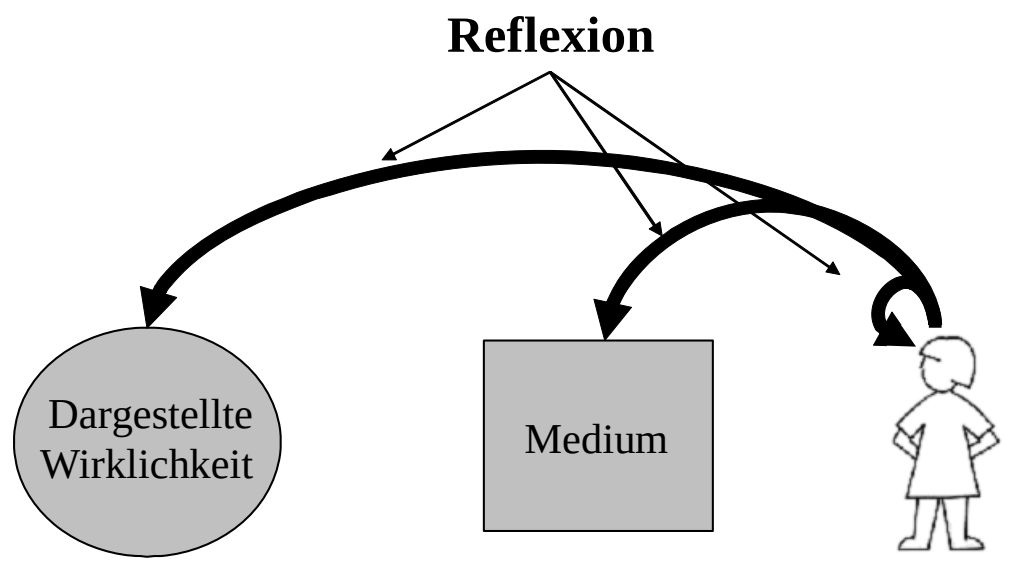

Die Proflexion ist das voranschreitende „systembewegende Moment“, das die Weiterentwicklung der Medienpädagogik bestimmt. Zuerst trifft die Proflexion der heranwachsenden Medien-Nutzer auf die zweidimensionale Fläche der Me- 
dien selbst, welche die Medien den Medien-Nutzern präsentieren. In der Nachfolge von Charles Sanders Peirce und William Morris unterscheide ich nun drei Analysearten, die syntaktische, die semantische und die pragmatische Analyse.

Die erste Analyseart wird infolgedessen die syntaktische Analyse genannt. Sie analysiert und interpretiert die zweidimensionale und formale Oberflächenstruktur der Medien. Die syntaktische Analyse thematisiert die Art und Weise, wie Sachverhalte und Situationen auf der Oberfläche der Medien dargestellt werden. Hier werden relativ formale Aussagen über die sinnliche Bestimmtheit dessen gemacht, was auf der Medienoberfläche erscheint. Die Syntaktik ist eine sinnlich-kognitive Wissenschaft, die vor allem Gestaltungsgesichtspunkte berücksichtigt. Wenn auf dem Monitor eines Computers z.B. ein Apfel abgebildet wird, dann fragt die Syntaktik, ob der Apfel als ein Ikon oder als eine gezeichnete Figur oder als ein Foto dargestellt wird. Die Darstellungsweise von Medieninhalten ist das Feld der Syntaktik. Weiterhin wird die Frage untersucht, ob der Apfel im Zentrum des Bildrahmens, am Bildrand, im Vordergrund oder im Hintergrund erscheint. Solche Fragen untersucht die Syntaktik. Es ist aber nicht die Aufgabe dieser Disziplin danach zu fragen, welche Situation z.B. auf dem Computermonitor sichtbar wird.

Schaubild 3: Die syntaktisch interpretierte Wirklichkeit als medienpädagogische Theorie

Erste Stufe der Medienpädagogik: Die syntaktische Analyse
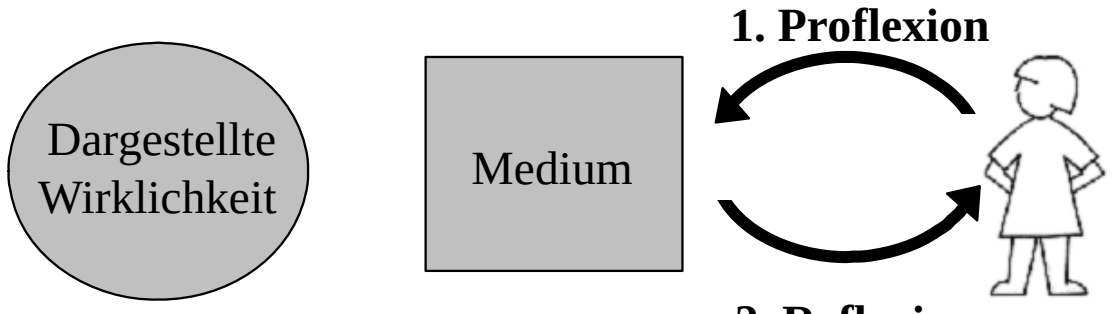

2. Reflexion

Zuerst trifft die Proflexion auf die zweidimensionale Fläche der Medien selbst, die auf dem Monitorfenster eines Computers erscheint. Dieses dem Medien-Nutzer vorgegebene flächige Material wird analysiert und interpretiert. Die Resultate der syntaktischen Analyse bezeichnen den Wissensbestand der Syntaktik, die weitgehend aus formalen Aussagen besteht und welche die Art und Weise der Medienpräsentation thematisieren.

Der Medien-Nutzer wird nun zum Medien-Analytiker. Seine Aufmerksamkeit wendet sich proflexiv und d.h. mit vorwärts ausgerichteter Blickrichtung dem Medium zu, analysiert die sinnliche Präsentationsform, beachtet vor allem figürliche Formen, also Figürliches oder Figuratives, formt Begriffe aus, fügt Begriffe zueinander und wendet sich sodann zurück d.h. reflektiert, um die gebildeten Kategorien und Begriffe reflexiv seinem kognitivem Wissensbestand einzuverleiben. 
Charakteristisch für die analysierende Tätigkeit ist immer das Zusammenspiel von Proflexion und Reflexion. Die Proflexion bezeichnet eine nach vorn hin ausgerichtete Tätigkeit, die sich dem Medium zuwendet. Wenn dieser Prozeß abgeschlossen ist, folgt der Proflexion die Reflexion - die eine nach rückwärts gerichtete geistige Tätigkeit darstellt, die Begriffe bildet und welche die neuen Erkenntnisse in seinen vormals aufgebauten Wissensbestand einfügt und sein Wissen hiermit verändert.

\subsection{Die Semantik als die zweite Stufe der aktiven Medienrezeption}

Alles, was dem Medien-Analytiker gegeben ist, zeigt sich auf der zweidimensionalen Oberfläche der Medien. Die Syntaktik erforscht die für sich reine Bildhaftigkeit der Medien, aber noch nicht das, was die auf dem Bildschirm sichtbaren Bilder darstellen. Anders ausgedrückt, ist das Thema der Syntaktik die figürliche Dimension der Medien. Das Figürliche verfügt aber über eine Referenz, die auf dargestellte Situationen hinweist. Wenn figürliche Konturen, Striche und vielfältig geformte zweidimensionale Figuren sich in Personen verwandeln, die in Situationen ihren Ort haben, dann ist der Übergang von der Syntaktik zur Semantik gelungen. Das Gebiet der Syntaktik ist überdies die zweidimensionale Fläche. Wenn aus farbigen Flächen und Konturen situierte Räume werden, dann befinden wir uns auf dem Gebiet der Semantik.

Die bildhafte oder die figürliche Wirklichkeit auf dem Monitorfenster ist niemals die ganze Wirklichkeit, die wir meinen, wenn wir Bilder betrachten. Um von der Syntaktik zur Semantik voranzuschreiten, überqueren wir eine Grenze, die zwischen den Medien als solchen und der von den Medien dargestellten Wirklichkeit verläuft. Die Proflexion bewegt sich gleichsam durch das Medium hindurch und folgt der Spur der Referenz, die auf vorgestellte Situationen hinweist. Ein Medium bringt etwas zum Ausdruck, das auf eine Situation hinweist, die sich außerhalb des Mediums befindet.

Schaubild 4: Die semantisch interpretierte Wirklichkeit als medienpädagogische Theorie

Zweite Stufe der Medienpädagogik: Die semantische Analyse

1. Proflexion

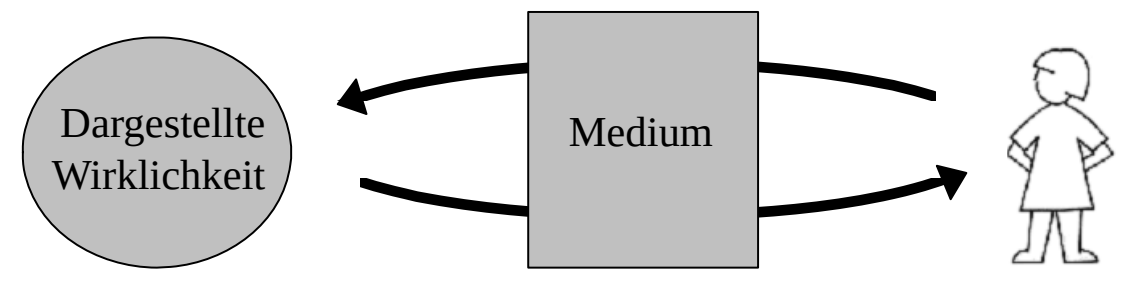

2. Reflexion 
Nehmen wir z.B. als Medium ein Photo von einem Baby, so weist das Photo als ein Bild über sich hinaus auf ein reales Baby, das auf dem Photo sichtbar wird. Die auf dem Photo sichtbare Figur des Babys wird von der Konturen des Baby-Bildes eingeschlossen. Alle diese Linien und Flächen sind Gegenstand der Syntaktik. Wenn es aber darum geht, aus der bildhaften Figur des Babys eine dreidimensionale Person des Babys werden zu lassen, die vielleicht in einem Kinderwagen liegt, dann ist diese dreidimensionale Vorstellung ein Gegenstand der Analyse und der Interpretation der Semantik. Die Ergebnisse dieser proflexiven semantischen Interpretation werden nun von der Reflexion eingeholt und zwar so, daß sich semantische Wissenszusammenhänge entwickeln können.

\subsection{Die Pragmatik als die dritte Stufe der aktiven Medienrezeption}

Der Übergang von der Semantik zur Pragmatik ist von besonderer Bedeutung, weil es sich hier um den Übergang von der wissenschaftlichen Theorie zur Medienpraxis handelt, die den Heranwachsenden mit den Medien über Handlungsprozesse verbindet. Die Grenze zwischen Wissenschaft und Praxis muß überwunden werden. In besonderer Weise ist diese Fragestellung für wissenschaftliche Disziplinen relevant, die dem Pragmatismus verbunden sind. Für den Pragmatismus sind wissenschaftliche Erkenntnisse nicht als solche relevant. Bedeutsam werden solche Erkenntnisse erst, wenn sie auf praktische Zusammenhänge bezogen werden können. Eine solche wissenschaftliche Disziplin ist die Medienpädagogik, für die theoretischen Medienerfahrungen erst eine bildende Bedeutung zugemessen werden kann, wenn die Pragmatik die Grenze von der Theorie zur Medienpraxis überschreitet und auf diese Weise den bildenden Erfahrungsbezug ermöglicht.

Der Pragmatismus, auf den ich mich beziehen möchte, ist mit Charles Sanders Peirce, Charles William Morris und John Dewey verbunden. John Dewey hat wiederholt darauf hingewiesen, daß die Pragmatik danach fragt, welche Bedeutung wissenschaftliche Erkenntnisse für praktische gesellschaftliche Lebenszusammenhänge haben. Die Pragmatik fragt also nach den „Konsequenzen“ oder nach den „Folgen“, die wissenschaftliche Satzzusammenhänge für das praktische Leben der zeichennutzenden Heranwachsenden haben.

Um den Übergang von der Semantik zur Pragmatik besser verstehen zu können, muß die Frage beantwortet werden, ob die Semantik in der Lage ist, die „ganze“ vorausgesetzte konkrete Wirklichkeit auszulegen. Wissenschaften können die individuelle und konkrete soziale Wirklichkeit niemals in ihrer vollen Singularität auslegen. Wissenschaften bewegen sich im Medium der Allgemeinheit und im Medium des Regelhaften, aber nicht im Medium des Einzelnen und des Individuellen. Die Wissenschaften können sich auf Einzelnes und Individuelles im Kontext der konkreten Praxis „beziehen“, sie können sich aber nicht im Medium des Konkreten bewegen. Nur praktische Handlungen können diese Anforderungen der konkreten Wirklichkeit erfül- 
len. Nur wenn wir praktisch handeln, stoßen wir auf Einzelnes und Singuläres. Begriffliche Zusammenhänge können zur konkreten Wirklichkeit nicht „durchstoßen“, sie können die konkrete Wirklichkeit allenfalls begrifflich zu fassen versuchen und Interpretationen ermöglichen.

Die Proflexion, die nun die Grenze zwischen der dargestellten Wirklichkeit und der konkreten situativen Praxis der Medien-Nutzer überschreitet, „meint“ die konkrete Ordnung der Dinge und Mitmenschen, mit denen wir im praktischen Handeln in Berührung kommen. In solchem praktischen Handeln sind uns konkrete Mitmenschen und einzelne Sachverhalte „unmittelbar“ gewiß.

Schaubild 5: Die Ergebnisse der syntaktischen und der semantischen Analyse und Interpretation werden auf die Situation des Lernenden bezogen

Dritte Stufe der Medienpädagogik: Die pragmatische Analyse

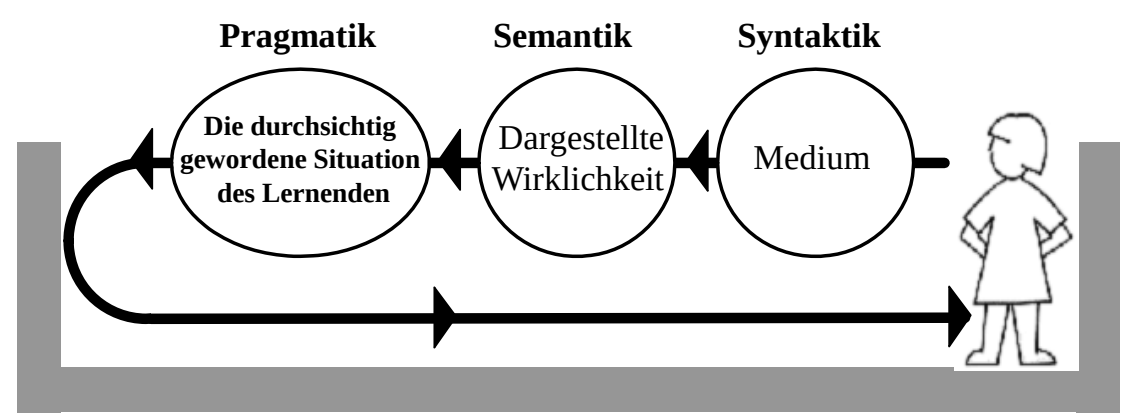

Die Pragmatik fragt nun nach der Bedeutung der Syntaktik und der Semantik für das praktische Handeln des Medien nutzenden Edukandus und ermittelt somit die Handlungsrelevanz der syntaktischen und der semantischen Wissenschaften. Das bedeutet, daß syntaktische und semantische Zusammenhänge nun auf das praktische Leben des Edukandus bezogen werden. Ich beziehe mich in diesem Kontext auf die Begriffe „Aufgegebenheit“ und „Gegebenheit“ von Fischer (1975). In der Wissenschaft ist uns die Wirklichkeit, auf welche die Wissenschaft sich bezieht, nur „gegeben“. In der Praxis ist uns die Wirklichkeit, die uns umgibt, aber „aufgegeben. Dies bedeutet, die praktische Wirklichkeit spricht uns an, damit wir uns handelnd zu ihr verhalten. Wissenschaftliche Zusammenhänge sind stets in Gefahr, ihre Handlungsrelevanz zu verlieren, weil sie nur feststellen können, was „ist“. Sie können uns aber nicht sagen, was wir tun sollen. Um diese Frage positiv beantworten zu können, ist der „Bezug“ zwischen Wissenschaft und der konkreten Wirklichkeit relevant.

Die Pragmatik als eine praktische Disziplin ist immer schon in konkreten Situationen verortet und betrachtet wissenschaftliche Ergebnisse vom Standpunkt der Praxis aus. Nur aus dieser Perspektive wird die Frage sinnvoll, 
welche die Handlungsrelevanz wissenschaftlicher Sätze erforscht. Erst die Pragmatik fragt nach der Bedeutung, die ein erfahrungsorientierter Bezug zwischen Wissenschaft und Praxis haben kann. Diesen Bezug zwischen Wissenschaft und Praxis bezeichnet Dewey als die edukative Funktion der Wissenschaft oder als die praktische Bedeutung, die wissenschaftliche Sätze haben können.

Im Kontext der Medienpädagogik haben wir uns gleichsam über drei Stufen hinweg bewegt. Dabei hat es sich gezeigt, daß wir das, was wir Wirklichkeit nennen, sich dreimal verwandelt hat. Auf der Vorstufe der Medienpädagogik ist uns die Medienwirklichkeit als eine sinnliche Wirklichkeit begegnet. Wir haben die Erfahrung gemacht, daß die Medienwirklichkeit für uns einen Erlebniszusammenhang darstellt.

Auf der ersten und auf der zweiten Stufe zeigte es sich, daß die Medienwirklichkeit als ein Theoriezusammenhang erscheint, der sich von der Syntaktik und der Semantik unterschiedlich, aber einander ergänzend darstellt. Die Medienwirklichkeit ließ den Erlebniszusammenhang hinter sich und verwandelte sich in einen Theoriezusammenhang. Wir konnten die Erfahrung machen, daß die Medienwirklichkeit, die uns umgibt, nicht mehr als ein erlebnishaftes Ereignis sich darstellte, sondern daß die Medienwirklichkeit gleichsam durchsichtig wurde und sich in einen begriffenen oder zumindest in einen begreifbaren Zusammenhang verwandelte.

Erst auf der dritten Stufe gelingt es der Pragmatik zum praktischen Handeln in der konkreten Wirklichkeit der Praxis „durchzustoßen“. Erst nun wird sichtbar, daß alle drei Stufen letztlich handlungsrelevant sein können. Wir haben erfahren, daß die uns umgebende Medienwirklichkeit zuerst erlebt, dann erkannt und zuletzt vom Gesichtspunkt der Handlungsrelevanz aus beurteilt werden muß. Dabei setze ich selbstverständlich voraus, daß Handlungsrelevanz im Kontext der Medienpädagogik pädagogische Handlungsrelevanz bedeutet.

Kommen wir am Schluß noch einmal auf die Frage nach der Medienwirklichkeit zurück, so ergibt sich, daß die Medienwirklichkeit auf der Vorstufe der Medienpädagogik als ein unmittelbarer Erlebniszusammenhang sichtbar wurde. Auf der ersten und auf der zweiten Stufe wurde deutlich, daß sich die Medienwirklichkeit als begriffene, kognitiv durchsichtige Medienwirklichkeit erwies. Auf der dritten Stufe der Medienpädagogik machen wir die Erfahrung, daß die Medienwirklichkeit von ihrem Fundament her einen Handlungszusammenhang darstellt, wobei die Medienwirklichkeit als Erlebniszusammenhang und theoretischer Zusammenhang zwei notwendige Voraussetzungen und Durchgangsstufen darstellen, die in der Medienwirklichkeit als Handlungszusammenhang enthalten sind. 


\section{Drei Phasen pädagogischer Medienproduktion, dargestellt am Beispiel einer Filmproduktion}

Das vorangegangene Kapitel macht deutlich, daß die wissenschaftlichen Disziplinen der Syntaktik, der Semantik und der Pragmatik in der Lage sind, die Prozesse der aktiven Medien- bzw. der Filmrezeption verständlich werden zu lassen. In diesem Kapitel über die medienpädagogischen Grundprobleme der Filmproduktion möchte ich zeigen, daß diese drei Analysearten geeignet sind, die medienpädagogischen Probleme der Medienproduktion bzw. der Filmproduktion einer adäquaten Lösung zuzuführen.

Verfolgen wir den Prozeß einer Filmproduktion von seinem Anfang bis zu seinem Ende, dann lassen sich drei Phasen der Medien- bzw. der Filmproduktion unterscheiden. Am Anfang des Produktionsprozesses steht die Medienidee bzw. die Filmidee, die damit auch die erste Phase der Medien- bzw. der Filmproduktion bezeichnet. In der zweiten Phase wird aus einer hoffentlich interessanten, aber abstrakten Medienidee bzw. der Filmidee ein medialer Handlungsplan bzw. ein differenziertes Drehbuch. In seinen Klassiker des Drehbuchschreibens erinnert Eugene Vale an Maurice Maeterlinck, der über das Drehbuchschreiben gesagt hat: „Ideen zu haben ist himmlisch, sie auszuarbeiten die Hölle“ (Vale 1988, S. 8; vgl. hierzu: Schäfer 1990). Den Abschluß der Medien- bzw. der Filmproduktion bildet die dritte Phase der Realisation des Medienprodukts bzw. der Arbeit mit der Kamera. Diese dritte Phase der Filmproduktion ist die eigentliche Drehphase, in der das Aufnahmeteam an wechselnden Drehorten eine Einstellung nach der anderen abdreht.

\subsection{Die syntaktische, semantische und pragmatische Dimension der Filmidee}

Charles William Morris hat die Semiotik, d.h. die Theorie der Zeichen im Anschluß an Charles Sanders Peirce weiterentwickelt und eine Konzeption vorgelegt, bei der die Semiotik aus den drei Teildisziplinen der Syntaktik, der Semantik und der Pragmatik besteht. Die Syntaktik begreift Morris als „formale Relation der Zeichen zueinander“ (Morris 1938, S. 8), wobei die Syntaktik zunächst von semantischen und pragmatischen Fragen absieht. Die Semantik untersucht „die Beziehung (oder die Referenz) zwischen den Zeichen und den Gegenständen, auf die sie anwendbar sind“ (Morris 1938, S. 213). Die Zeichen oder die Medien beziehen sich insofern auf Gegenstände und Menschen im Kontext von Situationen. Nach Auffassung von Morris ist die semantische Bedeutung eines Zeichens daher der bezeichnete Gegenstand oder der bezeichnete Sachverhalt. Situierte Gegenstände werden deshalb auch Referenzobjekte genannt. Die Semantik von Morris ist deshalb wesentlich eine Referenzsemantik. Was nun die Pragmatik betrifft, so untersucht sie die Beziehung zwischen den Zeichen und ihren Produzenten und ihren Rezi- 
pienten. Während also die Syntaktik die formale Struktur der Zeichen bzw. der Medien untersucht, thematisiert die Semantik die Beziehung der Zeichen bzw. der Medien zu den situierten Sachverhalten, die sie darstellen. Die Pragmatik schließlich ist die Wissenschaft, die den Ursprung, die Verwendung und die Zielbestimmung der Zeichen bzw. der Medien erforscht. Insofern ist die Pragmatik wesentlich die Wissenschaft von den Medienproduzenten und den Wirkungen von Zeichen bzw. von Medien auf die Medienrezipienten (vgl. Morris 1946, S. 365).

1993 habe ich mit einer Gruppe von Studenten, meinen beiden wissenschaftlichen Assistentinnen C. de Witt und H. Rudack und mit der technischen Unterstützung des Mediendidaktischen Zentrums der Universität Dortmund einen Film nach Texten des Philosophen und Pädagogen Fischer produziert. Die syntaktische Dimension der Filmidee besteht aus einem Gedanken, der sich in den Köpfen der Medienproduzenten, der Studenten, meiner Mitarbeiterinnen und bei mir allmählich ausbildet hat. Der Filmproduktion war ein Semester vorausgegangen, in dem ich mit den Seminarteilnehmern die Schriften von Fischer ein Semester lang interpretiert hatte. Man kann also nicht sagen, daß die Filmidee plötzlich da war. Sie ist erarbeitet worden und steht am Ende eines lang andauernden diskursiven Prozesses. Die Filmidee setzt sich zusammen aus den beiden Grundbegriffen, die sich wie ein roter Faden durch Fischers Werk ziehen: Die Proflexion und die Reflexion, das Voranschreiten und das Zurückweichen, die Voraus Wendung und die Zurückwendung, die Zuwendung zum anderen und die Abwendung von ihm, der Zusammenhang und das Gegenüber von Proflexion und Reflexion. Die Lektüre von Fischers Werken hat konkrete Bilder erzeugt. Dieser interpretierende Zeichenprozeß führt dazu, daß in den Köpfen der Beteiligten ein Gedanken entsteht, der aber bereits in literaler Form in den Schriften von Fischer immer wieder auftaucht und den Fischer selbst bereits „Proflexion“ und „Reflexion“ nennt (vgl. Fischer 1985).

Die Filmidee nimmt zunächst die Form einer gedanklichen Vorstellung an, die jederzeit verschriftlicht werden kann. Wenn dies geschieht, wird aus der zunächst oralen Filmidee eine literale Form, die gemäß den Regeln der Syntaktik analysiert werden kann.

In der formalen Bezeichnung „Proflexion und Reflexion“ kommt die gedankliche Form der Filmidee zum Ausdruck, wobei von der semantischen und der pragmatischen Bedeutung zunächst noch abstrahiert wird. Diese bloß syntaktische Form tritt möglicherweise in doppelter Gestalt auf. In ihrer oralen Form besteht sie lediglich aus semantisch bedeutungslosen Schallwellen, die aber phonetisch wohlgestaltete Lautreihen sind, die eine syntaktischmorphologische Bedeutung haben. In ihrer literalen Form nimmt die syntaktisch ausgerichtete Filmidee die Gestalt von semantisch unverstandenen Buchstabenreihen an, die einem bestimmten Schrifttyp des Computers wie z.B. Times New Roman verpflichtet sind. Beide syntaktischen Formen der Filmidee entsprechen durchaus dem Erfahrungs- und Kenntnisstand einiger Studenten zu Beginn der Filmproduktion. 
Wenn nun in der Seminardiskussion der Filmidee herausgearbeitet wird, daß die semantische Bedeutung von „Proflexion“ auf „Vorwärtsbewegung“ und „soziale Zuwendung“ und daß „Reflexion“ auf „Rückwärtsbewegung“ und „soziale Abwendung“ hinweist und daß weiterhin diese Grundidee auf den Dimensionen inhaltsorientierter und beziehungsorientierter praktischer Handlung, der emotionalen Dimension, der kommunikativen Dimension und der kognitiven Dimension ausdifferenziert werden muß, dann wird die Leistungsfähigkeit der Semantik der Filmidee den Studenten immer deutlicher bewußt.

Die pragmatische Analyse liefert sodann die entscheidenden Kriterien, die über die Annahme oder die Ablehnung dieser Filmidee entscheiden. Die pragmatische Dimension der Filmidee kommt zum Ausdruck, wenn nach der medienpädagogischen Bedeutung dieser Filmidee und dem zu drehenden Film für die studentischen Filmproduzenten, die an dem Prozeß dieser Filmproduktion beteiligt sind, und wenn nach der medienpädagogischen Bedeutung zukünftiger Rezipienten dieses Films gefragt wird. Die pragmatische Analyse erhält in dieser Phase die Form einer medienpädagogischen Antizipation oder einer Vorwegnahme des möglichen Erfolgs bzw. Mißerfolgs der Filmidee bei den Filmproduzenten und den Filmrezipienten.

\subsection{Die syntaktische, semantische und pragmatische Dimension des Drehbuches}

Es genügt natürlich nicht, wenn die Filmidee theoretisch wohl fundiert und von den künftigen Rezipienten bzw. den Zuschauern des Films voraussichtlich akzeptiert werden wird. Bevor die dritte Phase der Filmarbeit, die Arbeit mit dem Filmteam, mit der Kamera, mit der Beleuchtung usw. beginnen kann, muß der Film zuerst und zunächst einmal „geschrieben“ werden. Die Filmidee muß nun in ein „Dreh-Buch“ transformiert werden. Der künftige Film nimmt nun die Form eines Buches an. Die syntaktische Form eines Dreh-Buches wird sichtbar, wenn irgend ein Buch aufgeschlagen wird. Genau wie jedes andere Buch besteht eine Drehbuchseite aus zwei Spalten oder zwei Seiten. Auf der linken Seite werden die visuellen Gehalte der Einstellungen notiert und auf der rechten Seite die akustischen Inhalte wie der Text für die Schauspieler, die Geräusche und Hinweise zur Musik niedergeschrieben. Schließlich erhalten alle Einstellungen eine Nummer. Die Durchnummerierung der Einstellungen dient der Übersicht und der Verständigung. „Wir drehen heute die 21. (Einstellung)“, ist eine geläufige Redewendung.

Das Dreh-Buch als Buchform ist also Gegenstand der syntaktischen Analyse. Die syntaktischen Formen treten hervor, wenn ein Drehbuch analysiert und geschrieben werden soll. Die syntaktische Dimension des Drehbuchs wird sichtbar, wenn der Drehbuchautor die visuellen Informationen auf der linken Seite und die akustischen Informationen auf der rechten Seite des Drehbuchs einträgt. Hierbei ist die literale Form der Darstellung zu beachten. 
Die literale Form des Schreibens verlangt, daß ein Text zeilenweise produziert wird, wobei im Abendland die Zeilen von links nach rechts geschrieben und gelesen werden.

Behandeln die Regeln der Syntaktik die Frage, wie und in welcher schriftlichen Form der Film im Drehbuch zum Ausdruck kommt, dann legt die Semantik des Drehbuchschreibens fest, welche Inhalte in welchen Szenen dargestellt werden. Alle inhaltlichen Darstellungsfragen bei der Filmproduktion sind semantische Probleme, die gelöst werden müssen. Im Drehbuch muß sehr exakt festgehalten werden, welche Kameraeinstellungen welche Situationen darstellen.

Die Pragmatik des Drehbuchschreibens wird deutlich, wenn sich der Drehbuchautor die grundsätzliche Frage stellt, ob es genügend Rezipienten für einen Film gibt, der mit solch einem Aufwand an Zeit und Ressourcen aller Art gedreht werden soll. Hinter jeder Zeile eines Drehbuchs verbirgt sich die pragmatische Frage nach den Erwartungen der Zuschauer. Ein Medienproduzent und ein Regisseur, der wissenschaftliche Filme dreht, ist aber nicht nur den studentischen Rezipienten verpflichtet, sondern eine ebenso große Bedeutung hat die Frage nach dem Anspruchsniveau wissenschaftlicher Aussagen. Im Kontext einer Medienpädagogik des Pragmatismus sind solche Fragen stets in den Kontext der Pragmatik mit eingebunden.

\subsection{Die syntaktische, semantische und pragmatische Dimension der Realisation des Films}

Filme unterscheiden sich von Gedanken als Formen der Vorstellung und von Dreh-Büchern als literale Formen durch eine neue mediale Form, die Bildform. Ebenso wie die Medienpädagogik des Pragmatismus bezieht sich der französische Philosoph und Medientheoretiker Gilles Deleuze in seinem Buch Kino 1 auf den amerikanischen Semiotiker und Logiker Charles S. Peirce, „weil er eine allgemeine Klassifizierung von Bildern und Zeichen erstellt hat, ohne Zweifel die vollständigste und vielgestaltigste“ (Deleuze 1989, S. 11). Filme sind aber nicht unbewegte Standbilder, sondern „Bewegungsbilder“, wie Deleuze im Untertitel seines Buches Kino 1 bemerkt. Im Amerikanischen werden Filme daher auch „Movies“ bewegte Bilder genannt. Die Bewegung ist nach Deleuze daher auch das wesentliche Kennzeichen von Filmen.

In dieser dritten Phase der Filmproduktion, der Realisation des Films, wird aus dem Drehbuch mit Hilfe des Kameramanns ein Film, der aus vielen Einzelbildern besteht. Filme rücken daher bewegte Bilder ins Zentrum, die nun nach den Regeln der Syntaktik, der Semantik und der Pragmatik untersucht werden sollen.

Weil für Filmbilder die Bewegung das entscheidende Kennzeichen ist, fragt die syntaktische Analyse nach der Art und Weise der Bewegung, die in Filmbildern sichtbar wird. Hierbei sieht die Syntaktik von der semantisch- 
inhaltlichen Seite der Bewegung ab und abstrahiert auch von der pragmatischen Bedeutung der bewegten Filmbilder. Für die syntaktische Analyse bleibt somit eine formale Bestimmung der möglichen Bewegungsarten erhalten. Deleuze unterscheidet daher im Anschluß an Peirce und Bergson drei Bildebenen, die drei Bewegungsarten entsprechen (vgl. Deleuze 1989).

Im Kontext der Filmpädagogik des Pragmatismus unterscheidet die syntaktische Analyse der Filmarbeit insofern drei Arten von Bewegung:

1. Die Ensemble-Bewegung,

2. die Einstellungs-Bewegung und

3. die Montage-Bewegung.

Auf die Frage, was ein Bild ist, antwortet Deleuze mit einer Theorie der „Kadrierung“. Der Rahmen, französisch le „cadre“, grenzt ein Bild von seiner Umgebung ab. Der Rahmen macht ein Bild erst zu einem Bild. Ohne einen Rahmen gibt es kein Bild. Ein Bild besteht aus einem Rahmen, der ein Bildfeld eingrenzt, das Deleuze ein „Ensemble“ nennt. Ein Ensemble ist also ein zweidimensionales Bildfeld, das von einem feststehenden Rahmen umgeben ist. Wenn Filmbilder durch eine Kamera erzeugt werden, entsteht ein Ensemble mit einem feststehenden Rahmen, der sich nicht bewegt, wenn eine sogenannte „starre oder unbewegte Kamera“ eingesetzt wird. Eine Kamera mit einem festen Standort, die weder zur Seite noch nach oben oder unten „bewegt“ wird, produziert also ein Ensemble oder ein Bildfeld, dessen Rahmen sich nicht bewegt. In einem solchen Ensemble kann es nur eine Art von Bewegung geben, die sich innerhalb des feststehenden Bildrahmens vollzieht, die Deleuze deshalb auch Ensemble-Bewegung nennt. Diese erste Bewegungsart ist vergleichbar mit den Bewegungen der Schauspieler in einem Theater. Die Bühne kann als ein feststehender Rahmen betrachtet werden und die einzige Art von Bewegung, die in einem Theater aus der Perspektive der Zuschauer möglich ist, vollzieht sich innerhalb dieses Rahmens. Eine filmische Einstellung macht in einem vergleichbaren Sinn eine Ensemble-Bewegung sichtbar, wenn sich eine Einstellung von einem „Schnitt“ zum nächsten Schnitt erstreckt, wobei die Kamera sich nicht bewegt. Ein Beispiel für eine solche Ensemble-Bewegung ist z.B. ein Reiter, der zuerst auf der rechten Bildseite erscheint, sich über die Mitte des Bildfeldes hinweg bewegt und der dann das Bildfeld über den linken Bildrand verläßt.

Die zweite Art von Bewegung bei der Arbeit mit der Kamera im Kontext der syntaktischen Analyse entsteht, wenn die Kamera aufhört, eine starre Kamera zu sein und beginnt, sich in eine „bewegte Kamera“ zu verwandeln. Der Kameramann führt nun z.B. einen Schwenk nach rechts durch, wobei der Bildrahmen bewegt und auf diese Art und Weise die neue Form der „Einstellungs-Bewegung“ erzeugt wird. Bei einem Schwenk nach rechts folgt die Kamera z.B. der Bewegung eines Reiters. Eine solche Einstellung enthält eine Ensemble-Bewegung und zusätzlich bewegt sich mit dem Rahmen des Bildfeldes auch das Bildfeld selbst, so daß die Einstellungsbewegung zwei Arten von Bewegung beinhaltet, die Bewegung innerhalb des Rahmens - al- 
so die Ensemble-Bewegung - und zusätzlich die Bewegung des Rahmens - also die Einstellungs-Bewegung.

Eine dritte Art von Bewegung ist die Montage-Bewegung. Wenn die Arbeit des Filmteams und des Kameramann beendet ist, muß der Film „geschnitten“ werden. Im Schneideraum werden die vielen einzelnen Einstellungen zu einem geordneten Ganzen „montiert“. Die Kunst eines Regisseurs besteht auch darin, aus den zahlreichen Einstellungen eine geordnete Abfolge zu montieren, die Regeln der zeitlichen Folge so zu beachten, damit in den Köpfen der Filmrezipienten, also der Zuschauer, eine vollständige „Geschichte“ entsteht, die die Zuschauer auch nachvollziehen können.

Die Semantik der praktischen Filmproduktion thematisiert alles, was zur Referenz der Filmbilder gehört. Die bewegten Filmbilder, die visuellen Filmbilder ebenso wie die Klangbilder und die Geräuschbilder, stellen Situationen dar, die Interesse wecken sollen. Sie beziehen sich auf Situationen, die Referenzobjekte sind. Die erste Aufgabe der Referenzsemantik der Filmbilder besteht darin, die inhaltliche Fülle der Filmthemen zunächst einmal zu klassifizieren. In diesem Zusammenhang folge ich Deleuze, der drei Filmgattungen unterscheidet:

1. Die Aktionsfilme,

2. die Affektfilme und

3. die mentalen Filme.

Diese drei Filmgattungen, die Gegenstand der Semantik der praktischen Filmarbeit sind, habe ich an anderer Stelle dargestellt (vgl. Schäfer 1995). Von besondere Bedeutung für eine medienpädagogische Semantik des Films über die Filmgattungen hinaus ist das sogenannte „Narrative“, der „plot“ oder die Filmgeschichte. Die Grundbegriffe, die zum gemeinsamen Bestand von pragmatistischer Medienpädagogik und Dramaturgie der Films gehören, heißen ZEIT als semantisch dargestellte Handlungsfolge im Film, VORWÄRTSBEWEGUNG; CHARAKTERISIERUNG; MOTIV; ABSICHT; ZIELBESTIMMUNG; ANTIZIPATION UND SPANNUNG (vgl. Schäfer 1990). Was nun die Pragmatik der Arbeit mit der Kamera und mit dem Filmteam betrifft, so fragt die pragmatische Analyse nach der medienpädagogischen Bedeutung des Films für den heranwachsenden Filmrezipienten, der im Kontext der praktischen Medienpädagogik möglicherweise zugleich an der Produktion des Films mitbeteiligt ist.

\section{Abschließende Perspektiven: Über den Zusammenhang von Medienrezeption und Medienproduktion bzw. von Filmproduktion und Filmrezeption}

Was nun die Filmpragmatik betrifft, so ist es sinnvoll, einen engeren von einem weiteren Begriff von Pragmatik zu unterscheiden. Die Pragmatik im engeren Sinn ist jene Wissenschaft, die sich von der Syntaktik und der Semantik 


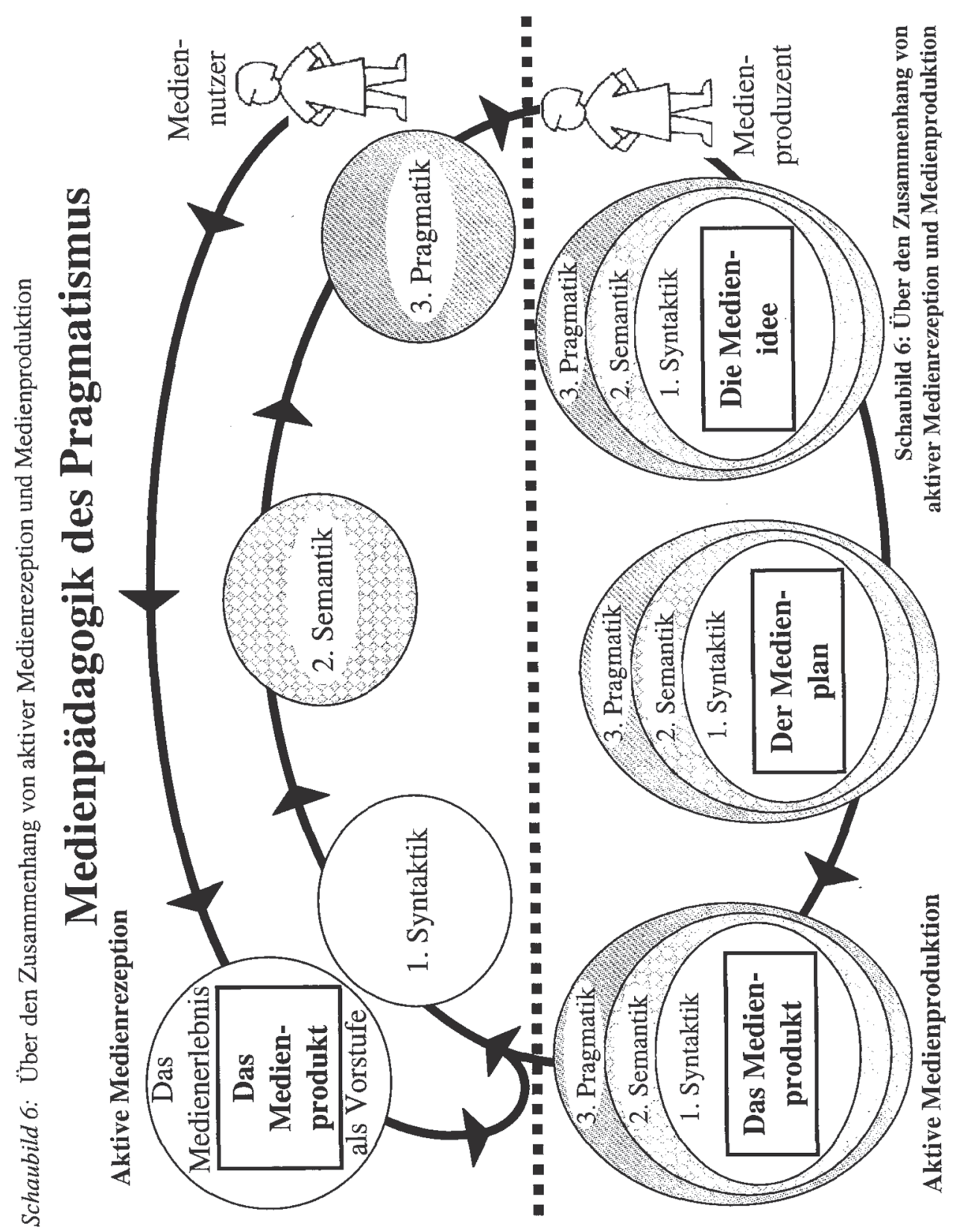


unterscheidet und die nach der Bedeutung filmischer Inhalte für die sich erweiternde, bildende Erfahrung von aktiven Medienrezipienten fragt. Die Pragmatik im weiteren Sinne umfaßt neben der aktiven Medienrezeption auch die Medienproduktion und fragt insofern nach dem Zusammenhang von Medienrezeption und Medienproduktion. Hierbei begreift sie diesen Zusammenhang gleichsam als einen kreisförmigen Prozeß, wobei sich Medienrezeption und Medienproduktion wechselseitig voraussetzen. Die Pragmatik in diesem weiteren Sinn untersucht den Ursprung der Medien im Medienproduzenten und die Wirkung der Medien auf den Medienrezipienten. Auf diese Art und Weise trifft sie eine Unterscheidung, die zwischen der Wirkung von syntaktischen Strukturen, der semantischen Bedeutung von Inhalten und den pragmatischen Sinngehalten von medial vermittelten Bildungsprozessen differenziert, die einen Beitrag für sich kontinuierlich weiterentwickelnde Erfahrungsprozesse zu leisten vermögen.

\section{Literatur}

Deleuze, G.: Das Bewegungsbild. Kino 1. Frankfurt a.M. 1989 (übersetzt von U. Christians und U. Bokelmann)

Dewey. J.: Syllabus: Types of Philosophie Thought. In: John Dewey: The Middle Works, Vol. 13:1983, S. 349-396

Dewey, J.: Logic: The Theory of Inquiry. In: John Dewey, The Later Works 1925-1953, Vol. 12:1938. Carbondale and Edwardsville 1986

Fischer, F.: Darstellung der Bildungskategorien im System der Wissenschaften. Ratingen Kastellaun 1975 (aus dem Nachlaß hrsg., eingeleitet und mit Nachworten versehen von D. Benner und W. Schmied-Kowarzik)

Fischer, F.: Logik der Menschlichkeit. Späte Schriften und letzte Entwürfe 1960-1970. Werkausgabe Band IV. Wien und München 1985 (hrsg. von M. Benedikt und W.W. Priglinger)

Morris, Ch. W.: Foundations of the Theory of Signs. Chicago 1938

Morris, Ch. W.: Signs, Language and Behavior. New York 1946

Nagl L: Charles Sanders Peirce. Frankfurt New York 1992

Nöth, W.: Handbuch der Semiotik. Stuttgart Weimar 2000 (2., vollständig neu bearbeitete und erweiterte Auflage mit 89 Abbildungen)

Schäfer, K.-U.: Eugene Vales, John Deweys und Franz Fischers Beiträge zu einer neuen Medienpädagogik. In: Norderstedter Hefte für Philosophie und Pädagogik, 5 (1990) 2, S. 48-77

Schäfer, K.-H.: Bilder, Bildung und Wirklichkeit. Eine Interpretation von F. Fischers „Geometrie des Menschlichen“, Teil I. In: Pädagogische Rundschau, 48 (März/April 1994)2, S. 155-184

Schäfer, K.-H.: Entwurf einer kommunikativen Filmtheorie. In: Zöllner, D. (Hrsg.): Wege zur Mitmenschlichkeit und Frieden. Franz Fischer-Symposion 1994. Norderstedt 1994, S. 91-112

Schäfer, K.-H.: Bildsinn und Bildungssinn bei Johann Arnos Comenius, Gilles Deleuze und Franz Fischer. In: Norderstedter Hefte für Philosophie und Pädagogik, 1995/1

Schäfer, K.-H.: Medienpädagogik als Medienmethodik. In: Fischer-Buck, A./Schäfer, K.- H./Warzel, A./Zöllner, D. (Hrsg.): Sinn und Wirklichkeit. Franz Fischer Jahrbücher für Philosophie und Pädagogik, 1996, S. 56-77 (online verfügbar unter http://www.fbl2. uni-dortmund.de/poison/) 
Schäfer K-H.: „Der Mann, der im Nacken keine Augen hatte“ - Ein Film nach Texten von Franz Fischer. Bericht von A. Fischer-Buck. In: Norderstedter Hefte für Philosophie und Pädagogik, 9 (1994) 1, S. 46-68

Schäfer K.-H.: Homer und Platon als Gründungsväter der Medienpädagogik. In: Fischer- Buck, A./ Warzel, A./Schäfer, K.-H./Zöllner, D. (Hrsg.): Franz-Fischer Jahrbuch für Philosophie und Pädagogik, 1997, S. 75-95 (online verfügbar)

Schäfer K.-H.: Mediensemiotik und Medienpädagogik. In: Fischer-Buck, AVSchäfer, K.- H./Zöllner, D. (Hrsg.): Werte II, Humanität und Wissenschaft. Franz-Fischer Jahrbuch für Philosophie und Pädagogik, 1998 (online verfügbar)

Schäfer, K.-H.: 10 Thesen zur kommunikativen Computerpädagogik. In: Fischer-Buck, A/Schäfer, K.-H./Zöllner, D. (Hrsg.): Franz-Fischer Jahrbuch für Philosophie und Pädagogik, 1999 (online verfügbar)

Schäfer K.-H.: Medienpädagogik als Teildisziplin der Allgemeinen Erziehungswissenschaft. In: Aufenanger, St./Schulz-Zander, R./D. Spanhel (Hrsg.): Jahrbuch Medienpädagogik 1, Opladen 2000 (online verfügbar)

Vale, E.: Die Technik des Drehbuchschreibens für Film und Fernsehen. München 1988 de Witt, C.: Neue Medien und die Pädagogik des Pragmatismus. Strukturen Neuer Medien und die Notwendigkeit neuer Formen von Erfahrung in der Pädagogik. Dortmund 2000 (Habil.-Schrift, noch unveröffentl. Manuskript) 